\title{
NeuroImage
}

ELSEVIER

\section{Investigating the post-stimulus undershoot of the BOLD signal- A simultaneous fMRI and fNIRS study}

\author{
Matthias L. Schroeter, ${ }^{\text {a,b,* }}$ Thomas Kupka, ${ }^{\text {a }}$ Toralf Mildner, ${ }^{\text {a }}$ \\ Kâmil Uludağ, ${ }^{c}$ and D. Yves von Cramon ${ }^{\mathrm{a}}$ \\ ${ }^{\mathrm{a}}$ Max-Planck-Institute for Human Cognitive and Brain Sciences, Stephanstr. 1A, Germany \\ ${ }^{\mathrm{b}}$ Day Clinic of Cognitive Neurology, University of Leipzig, Liebigstr. 22A, 04103 Leipzig, Germany \\ 'fMRI Center of the Department of Radiology, University of California San Diego, 9500 Gilman Drive/Osler Lane, La Jolla, CA 92039-0677, USA
}

Received 14 June 2005; revised 26 August 2005; accepted 19 September 2005

Available online 27 October 2005

\begin{abstract}
Measuring the hemodynamic response with functional magnetic resonance imaging (fMRI) together with functional near-infrared spectroscopy (fNIRS) may overcome limitations of single-method approaches. Accordingly, we measured the event-related hemodynamic response with both imaging methods simultaneously in young subjects during visual stimulation. An intertrial interval of $60 \mathrm{~s}$ was chosen to include the prolonged post-stimulus undershoot of the blood oxygenation level dependent (BOLD) signal. During visual stimulation, the BOLD signal, oxy-, and total hemoglobin $(\mathrm{Hb})$ increased, whereas deoxy-Hb decreased. The post-stimulus period was characterized by an undershoot of the BOLD signal, oxy-Hb, and an overshoot of deoxy-Hb. Total $\mathrm{Hb}$ as measured by fNIRS returned to baseline immediately after the end of stimulation. Results suggest that the post-stimulus events as measured by fNIRS are dominated by a prolonged high-level oxygen consumption in the microvasculature. The contribution of a delayed return of blood volume to the BOLD post-stimulus undershoot in post-capillary veins as suggested by the Balloon and Windkessel models remains ambiguous. Temporal changes in the BOLD signal were highly correlated with deoxy-Hb, with lower correlation values for oxy- and total $\mathrm{Hb}$. Furthermore, data show that fNIRS covers the outer $1 \mathrm{~cm}$ of the brain cortex. These results were confirmed by simultaneous fMRI/fNIRS measurements during rest. In conclusion, multimodal imaging approaches may contribute to the understanding of neurovascular coupling.
\end{abstract}

(C) 2005 Elsevier Inc. All rights reserved.

Keywords: BOLD signal; Near-infrared spectroscopy; Post-stimulus undershoot

\footnotetext{
* Corresponding author. Max-Planck-Institute for Human Cognitive and Brain Sciences, Stephanstr. 1A, 04103 Leipzig, Germany. Fax: +49 34199 40221.

E-mail address: schroet@cbs.mpg.de (M.L. Schroeter).

Available online on ScienceDirect (www.sciencedirect.com).
}

\section{Introduction}

Several recent studies compared the hemodynamic response to brain activation as measured by functional near-infrared spectroscopy (fNIRS) with another imaging method, such as functional magnetic resonance imaging (fMRI) (Hoge et al., 2005; Kennan et al., 2002; Kleinschmidt et al., 1996; Mehagnoul-Schipper et al., 2002; Obrig et al., 2000b; Seiyama et al., 2004; Siegel et al., 2003; Strangman et al., 2002; Toronov et al., 2001a,b, 2003; Wenzel et al., 2000) or positron emission tomography (PET) (Hock et al., 1997; Villringer et al., 1997). While optical measurements are poorer in spatial resolution and depth penetration than fMRI, they are biochemical specific and, consequently, provide information about changes in oxy-, deoxy-, and total hemoglobin $(\mathrm{Hb})$ with a high temporal resolution (Hoshi, 2003; Villringer and Chance, 1997). Moreover, it was suggested that fNIRS is particularly sensitive to the microvasculature (Boushel et al., 2001; Cannestra et al., 2001) because, for larger vessels such as arteries and veins, entering light cannot escape (Liu et al., 1995a,b). The blood oxygenation level dependent (BOLD) signal represents, on the other hand, all spatial scales of venous vessels (Lee et al., 2001; Strangman et al., 2002; Weisskoff, 1999). These advantages of fNIRS can be used to better understand the nature of the hemodynamic response to neuronal activation and the origin of the BOLD signal. On the other hand, optical studies are limited because anatomical information is not obtained. Simultaneously acquired optical and fMRI data may synergize and overcome this limitation as the MRI data provide information about the location of the optical probes.

The present study aimed at comparing the temporal behavior of the hemodynamic response between fMRI and fNIRS in the visual cortex of young healthy humans. All previous studies that combined fNIRS with another imaging method did not investigate the post-stimulus period of the hemodynamic response. To include that period, we chose a long intertrial interval of $60 \mathrm{~s}$ (Mildner et al., 2001). An event-related approach was employed 
to detect the hemodynamic response to a single trial (Burock et al., 1998; Schroeter et al., 2002, 2004c,d). Measurements were performed simultaneously with fMRI and fNIRS.

\section{Materials and methods}

\section{Subjects}

Twelve healthy subjects participated in the study (righthanded, mean age 24.8 \pm 1 years, range 24-27, 4 female). Written informed consent was obtained from all subjects after complete description of the study to the subjects prior to the session. The research protocol was approved by the ethics committee of the University of Leipzig and was in accordance with the latest version of the Declaration of Helsinki. All subjects had normal or corrected-to-normal vision and normal color vision.

\section{Visual stimulation}

For task-induced activation, a simple visual task was employed. During periods of control, subjects had to watch a small gray fixation cross-positioned in the center of a black screen. To focus attention, they had to press a button each time they saw a small black hole appearing in the center of the fixation-cross at randomized time intervals. During periods of stimulation, a $12 \times 14$ array of red L-shapes randomly rotating at a frequency of $8 \mathrm{~Hz}$ on the black background was presented as a strong full-field visual stimulus, while the same attention task had to be performed as during the control periods. The duration of the visual stimulus was $6 \mathrm{~s}$, and the recovery period after each stimulus was $54 \mathrm{~s}$. Ten complete cycles of visual stimulation were recorded. Furthermore, fMRI and fNIRS data were obtained during a resting condition of 10 min (closed eyes). Four blocks (visual stimulation-rest-visual stimulation-rest) resulted in a total time of $40 \mathrm{~min}$.

\section{Functional near-infrared spectroscopy}

Changes in the concentration of oxy-, deoxy-Hb, and the redox state of the cytochrome-c-oxidase (Cyt-Ox) were measured by an NIRO-300 spectrometer (Hamamatsu Photonics K.K., Japan) and expressed in nanomolars. Values were calculated according to Cope and Delpy (1988). Moreover, we calculated changes in the concentration of total $\mathrm{Hb}$ (sum of oxy- and deoxy$\mathrm{Hb}$ ) as a measure for changes in regional cerebral blood volume (rCBV). Changes in the redox state of Cyt-Ox may be biased by cross talk, that is, a change in $\mathrm{Hb}$ might yield an artifactual change in Cyt-Ox (Heekeren et al., 1999; Uludag et al., 2002). Because continuous spectra approaches are recommended for Cyt-Ox and the NIRO-300 uses only four wavelengths $(775,810$, 850 , and $910 \mathrm{~nm}$ ), we report changes in Cyt-Ox without discussing it. The NIRO-300 allows parallel measurement of two channels at a sampling frequency of $6 \mathrm{~Hz}$. The emitterdetector spacing was $4 \mathrm{~cm}$, and a differential pathlength factor of 6.26 was used (Duncan et al., 1995). Two emitter-detector pairs were placed crosswise; one was oriented vertically, the other horizontally (Fig. 1). The point of intersection between both emitter-detector pairs was localized at position $\mathrm{O} 1$ of the international 10/20 (electroencephalography) system over the left visual cortex (Fig. 2; Homan et al., 1987; Okamoto et al., 2004). To enable simultaneous measurements with fMRI, light was transmitted via approximately $12 \mathrm{~m}$ long fiber cables (Hamamatsu Photonics K.K., Japan) and transformed outside the room containing the fMRI scanner.

Firstly, time courses of the different chromophores were averaged between the horizontally and the vertically oriented emitter-detector pairs for each subject to improve the signal-tonoise ratio and resulting in a sampling volume centered below the two emitter-detector pairs. Thereafter, time courses were resampled to a frequency of $1 \mathrm{~Hz}$ and, for the visual stimulation, averaged over the several stimulation cycles and across subjects. For statistical analysis, the mean concentration changes from 'baseline' ( -6 to $-1 \mathrm{~s}$ before stimulation onset) to the hemodynamic response in the 'stimulus period' (7-13 s after stimulation onset) and 'post-stimulus period' (19-33 s after stimulation onset) were calculated for each subject by averaging the time courses in these intervals (Fig. 3). Significance values were calculated by Student's $t$ tests against 0 . The distance between skin and cortical surface was measured at the point of intersection between both emitter-detector pairs (position $\mathrm{O} 1$ of the international 10/20 system over the calcarine sulcus; Homan et al., 1987) in standard high-resolution whole-head 3D MRI data sets (3.0 T Medspec Scanner, Bruker, Germany; T1weighted modified driven equilibrium Fourier transform [MDEFT] protocol [Lee et al., 1995; Norris, 2000; Ugurbil et al., 1993], 128 sagittal slices, $256 \times 256$ matrix, field of view $25.0 \times 25.0 \times 19.2 \mathrm{~cm}, 1.5 \mathrm{~mm}$ slice thickness) interpolated to an isotropical resolution of $1 \mathrm{~mm}$ using a fourth-order b-spline method (Thevenaz et al., 2000). The mean skin-cortex distance, averaged across subjects, was $11.1 \pm 2.2 \mathrm{~mm}$ in accordance with Okamoto et al. (2004).

\section{Functional magnetic resonance imaging}

fMRI experiments were performed using the $3.0 \mathrm{~T}$ whole-body scanner as described above. Anatomical images were obtained with the MDEFT sequence as mentioned above. For functional imaging, an echo planar imaging (EPI) sequence with a $64 \times 64$ matrix, a field of view of $192 \mathrm{~mm}$, and a repetition time of $1 \mathrm{~s}$ was used (acquisition bandwidth $100 \mathrm{kHz}$, echo train length $41 \mathrm{~ms}$, echo time $30 \mathrm{~ms}$ ). Optodes were visualized by vitamin $\mathrm{E}$ capsules on individual MRIs (Fig. 2). Twelve oblique slices (thickness $4 \mathrm{~mm}$, gap $1 \mathrm{~mm}$ ) were oriented parallel to the plane spanned between the two emitter-detector pairs (Fig. 1).

fMRI data were processed as follows: the image time series was first corrected for bulk motion using a motion correction routine incorporated in the Leipzig Image Processing and Statistical Inference Algorithms (LIPSIA) software package developed at the Max-Planck-Institute for Human Cognitive and Brain Sciences (Lohmann et al., 2000). A high-pass filter $(0.0083 \mathrm{~Hz})$ was applied in order to remove baseline drifts. The square between the two emitter-detector pairs was defined as the region of interest (ROI) in every slice (Fig. 1). The ROI had the same extension in all slices, avoiding any assumption on depth penetration of nearinfrared light and allowing a specific analysis for each slice separately. It was located at the visual cortex as proved by individual anatomical MRI. Signal changes in the ROI were averaged between all voxels for every slice separately. For the visual stimulation, resulting time courses were averaged between the ten stimulation cycles and across subjects. Again, the mean 


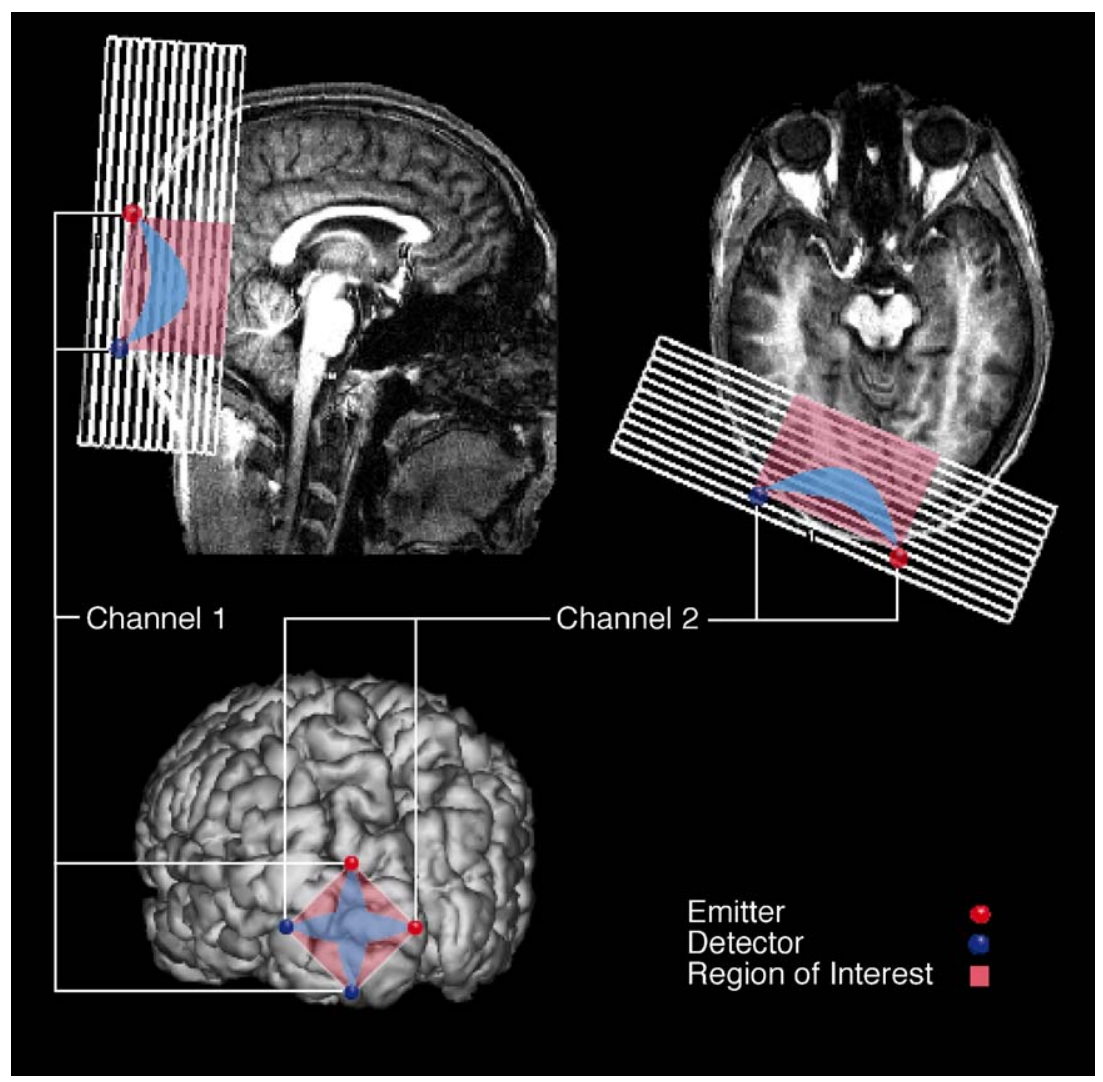

Fig. 1. Sampling volume (blue) between the two emitter-detector pairs of fNIRS in relation to the slices of fMRI (white). Emitter-detector pairs of fNIRS were placed crosswise, one oriented vertically, the other horizontally. The point of intersection was localized at position $\mathrm{O} 1$ of the international $10 / 20$ (electroencephalography) system over the left visual cortex. The fMRI slices were oriented parallel to the plane spanned between the four optodes. The BOLD signal was analyzed for each slice in the respective region of interest. Highest correlation between oxy-/deoxy-hemoglobin and the BOLD signal is expected for the slice in the center of the banana-shaped fNIRS sampling volume. Note that illustration is schematic only.

concentration changes of the BOLD signal from 'baseline' to the hemodynamic response in the 'stimulus period' and 'post-stimulus period' were calculated for each subject in the averaged time courses. Significance values were calculated by Student's $t$ tests against 0 .

To visualize activated brain regions in one subject, activated voxels were selected by correlating the time course of each voxel with a design function representing the characteristics of the stimulus (Fig. 2A). The time points of the design function from 6 to $12 \mathrm{~s}$ after stimulation onset were referred to as the activated state, and the last $25 \mathrm{~s}$ of the resting period were referred to as the resting state. Other time points during the transition period between the activated and the resting states were ignored, avoiding any assumptions for the post-stimulus period. Furthermore, the time course of the concentration change of deoxy-Hb was used as a design function in the same subject (Fig. 2B). Results are generally reported as mean $\pm \mathrm{SD}$, if not stated otherwise.

\section{Results}

All subjects sustained their attention during visual stimulation $(496.8 \pm 40.8 \mathrm{~ms}$ mean reaction time and $4 \pm 3.5 \%$ mean error rate). As illustrated in Fig. 2A for one subject by correlation with a boxcar function, visual stimulation led to an activation in the visual cortex bilaterally around the calcarine sulcus. If the time course of deoxy-Hb as measured by fNIRS was used as a design function, the same area was activated, although with slightly lower correlation coefficients presumably due to random fluctuation of the optical properties in the non-brain slices in the sampling volume of the near-infrared light (Fig. 2B). Visual stimulation led to a significant increase of oxy- $\mathrm{Hb}$, total $\mathrm{Hb}$, and Cyt-Ox right after stimulation onset (Fig. 3, Table 1). Furthermore, deoxy-Hb decreased significantly. The BOLD signal increased in each slice (Table 1), when the signal was averaged in the square between the two emitter-detector pairs as an ROI. The hemodynamic response peaked $11 \mathrm{~s}$ and reached baseline values roughly $15 \mathrm{~s}$ after stimulation onset (Fig. 3).

During the post-stimulus period, oxy-Hb decreased below, whereas deoxy-Hb increased above baseline values (Fig. 3, Table 1). Concentration of total $\mathrm{Hb}$ and the redox state of the Cyt-Ox did not change significantly during this period. The BOLD signal decreased below baseline values. The post-stimulus hemodynamic response peaked roughly at $23 \mathrm{~s}$ and reached baseline values about $40 \mathrm{~s}$ after stimulation onset (Fig. 3).

To estimate signal-to-noise ratios across subjects, variation coefficients were calculated as standard deviation divided by mean values as reported in Table 1 (Bortz, 1999). Regarding optical parameters, signal-to-noise ratio was generally highest for deoxy-Hb compared with oxy- $\mathrm{Hb}$, Cyt-Ox, and total $\mathrm{Hb}$ (for the stimulus period: $0.57,1.02,1.07,2.46$; for the post-stimulus period: $1.42,1.92,8.46,5.57$, respectively; calculated according to Bortz, 1999). Obviously, changes during the stimulation 


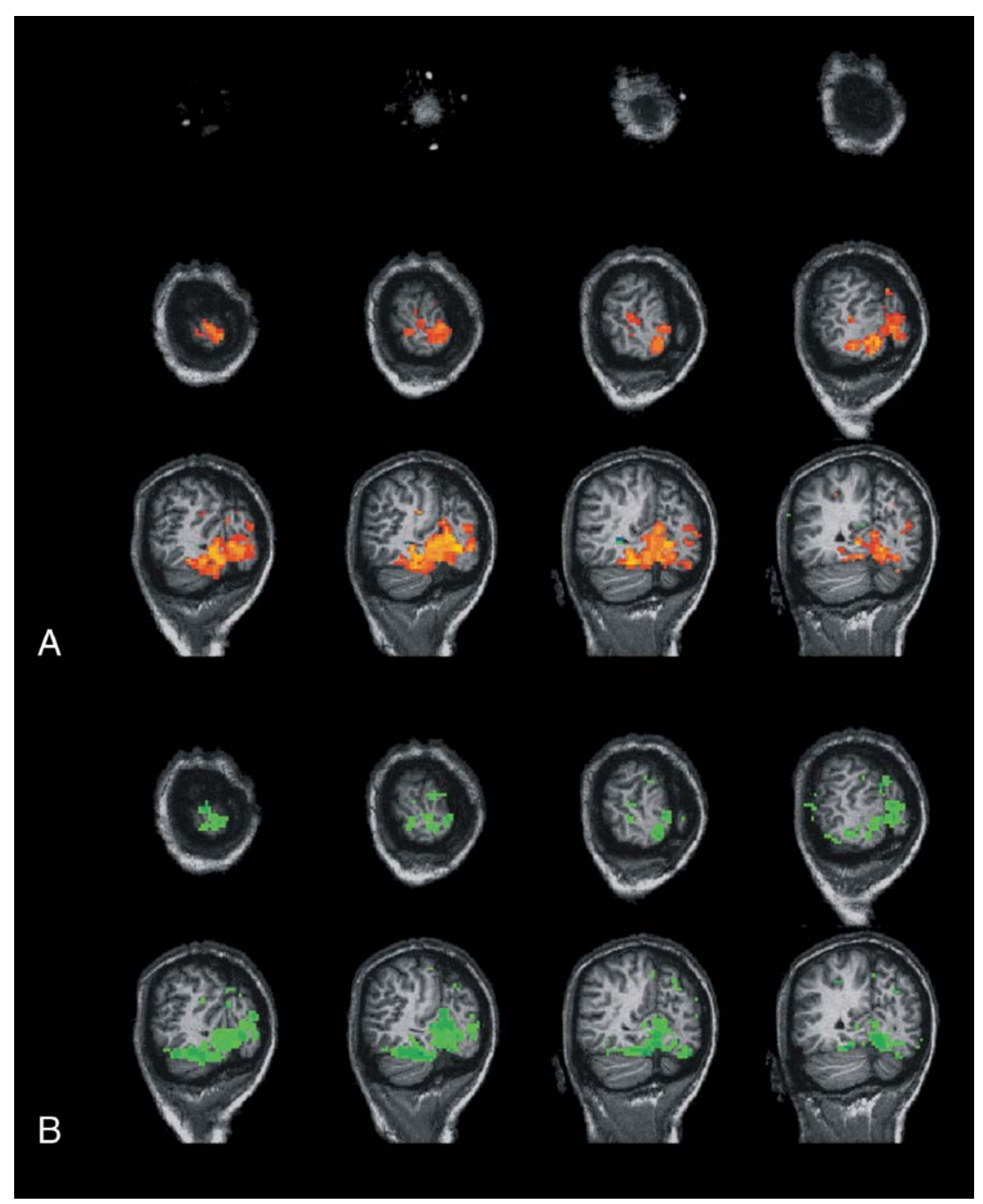

Fig. 2. Averaged correlation maps for one subject. BOLD contrast. (A) If a boxcar function (not convolved with a hemodynamic response function and shifted by $6 \mathrm{~s}$ ) was used as a design function $(0.85 \leq r \leq 0.95)$. (B) If the time course of deoxy-hemoglobin as measured by fNIRS was used as a design function $(0.8 \leq r \leq 0.83)$. Optode positions are marked by vitamin E capsules (see first row in panel A).

period had a higher signal-to-noise ratio than post-stimulus changes, which was also the case for the BOLD signal (variation coefficient for the stimulus period 0.38 , for the post-stimulus period 0.87). Therefore, it may be concluded that the poststimulus period had a higher inter-individual variability than the stimulus period in agreement with Mildner et al. (2001).

To analyze depth penetration of fNIRS, we correlated the eventrelated time courses for deoxy-Hb with the BOLD time courses individually for each slice and subject during visual stimulation (11 $\mathrm{s}$ before to $48 \mathrm{~s}$ after stimulation onset). Deoxy-Hb was chosen because the BOLD signal arises mainly from this chromophore (Buxton et al., 1998; Ogawa et al., 1993). Furthermore, a partial correlation was calculated adjusted for concentration changes of total $\mathrm{Hb}$ as the BOLD signal is influenced by changes of $\mathrm{rCBV}$ according to Ogawa et al. (1993) and the Balloon model (Buxton et al., 1998). Individual $r$ values were normalized with a Fisher's $Z$ transformation before averaging. As illustrated in Fig. 4, normalized Pearson correlation coefficients differed significantly between the several slices during visual stimulation (repeated measure ANOVA $d f=8, F=8.16, P<0.001)$. The highest correlation was found for a depth of $15 \mathrm{~mm}$, which was significantly higher than in the adjoining slices. Adjustment for changes of total $\mathrm{Hb}$ led again to significant differences between the several slices $(d f=8, F=$ 8.76, $P<0.001)$ and, overall, increased correlation coefficients compared with non-adjusted $Z$ values. Correlation of oxy-Hb with the BOLD signal was lower than that of deoxy-Hb, reaching a maximal $Z$ value of $0.69 \pm 0.54$ in a depth of $20 \mathrm{~mm}$ and showing the same depth dependence like deoxy-Hb (not shown). Correlation was lowest for total $\mathrm{Hb}$ and Cyt-Ox (maximal $Z$ value $0.31 \pm$ $0.29,0.41 \pm 0.38$, respectively).

To analyze whether correlation values were different during the stimulus and post-stimulus periods, we repeated the correlation analysis as described above for both periods separately (stimulus period $0-15 \mathrm{~s}$, post-stimulus period $15-48 \mathrm{~s}$ after stimulation onset). The analysis was limited to the slice $15 \mathrm{~mm}$ below the optode layer because this slice showed the highest correlation between chromophores and the BOLD signal (see Fig. 4). As illustrated in Table 2, (absolute) mean correlation coefficients were higher for the stimulus period compared with post-stimulus events (oxy-Hb $P<0.01$; deoxy-Hb and Cyt-Ox $P<0.001$; total Hb not significant; 1-tailed paired Student's $t$ tests). Furthermore, (absolute) mean correlation coefficients were highest for deoxy-Hb in 

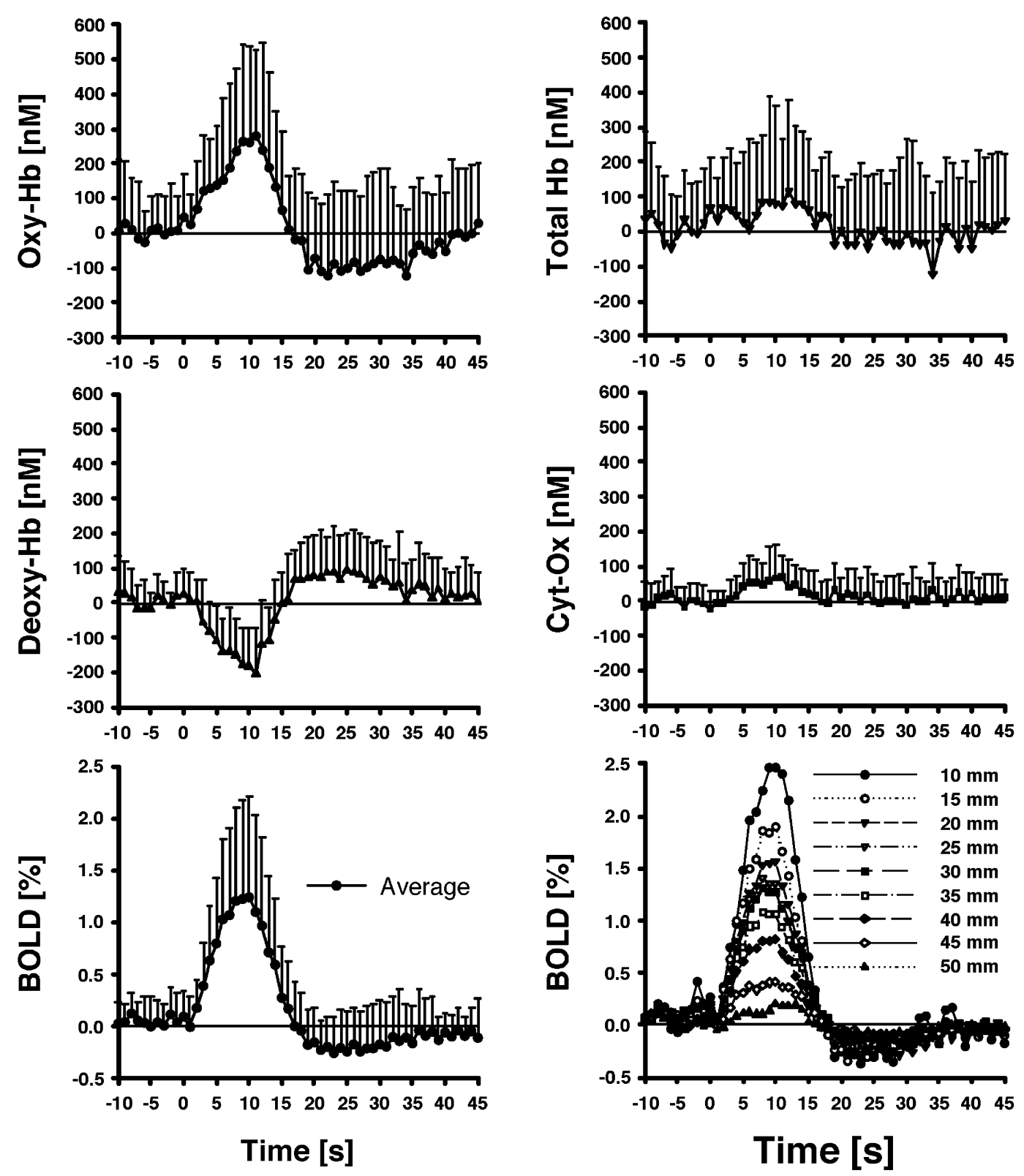

Fig. 3. Concentration changes of oxy-, deoxy, total hemoglobin (Hb), and cytochrome-c-oxidase (Cyt-Ox) as measured by fNIRS and the BOLD signal as measured by fMRI (time courses for the square area between the four optodes, averaged over all measured slices and for every slice separately). Visual stimulation started at $0 \mathrm{~s}$ and continued until $6 \mathrm{~s}$. Mean $\pm \mathrm{SD}$.

comparison with all other chromophores (stimulus period: deoxy$\mathrm{Hb}$ vs. oxy-Hb $P<0.01$, vs. total $\mathrm{Hb}$ and Cyt-Ox $P<0.001$; poststimulus period: deoxy-Hb vs. oxy-Hb $P<0.1$, vs. total $\mathrm{Hb}$ and Cyt-Ox $P<0.005)$. Correlation coefficients were higher for oxy$\mathrm{Hb}$ than total $\mathrm{Hb}$ (stimulus period: $P<0.001$; post-stimulus period: $P<0.005)$ and higher for Cyt-Ox than total $\mathrm{Hb}$ during the stimulus period $(P<0.01)$.

Moreover, we correlated the individual total time courses for deoxy-Hb and the BOLD signal during rest (closed eyes). As during stimulation, correlation coefficients differed between the several slices $(d f=8, F=4.13, P<0.001)$, with highest values in a depth of $15 \mathrm{~mm}$ (Fig. 4). Adjusting for changes in total $\mathrm{Hb}$ led once more to significant differences between slices $(d f=8, F=3.94, P<$ $0.001)$ and elevated correlation coefficients. Correlation coefficients of oxy-Hb, total $\mathrm{Hb}$, and Cyt-Ox with the BOLD signal were again lower than those of deoxy-Hb (maximal $Z$ values of $0.16 \pm$ $0.18,0.12 \pm 0.16$, and $0.07 \pm 0.1$, respectively).

\section{Discussion}

During stimulation, the BOLD signal, oxy-, total $\mathrm{Hb}$, and Cyt$\mathrm{Ox}$ increased, whereas deoxy-Hb decreased in accordance with recent studies (Buxton et al., 1998; Heekeren et al., 1999; Obrig et al., 2000b; Schroeter et al., 2004a; Seiyama et al., 2004; Wobst et al., 2001), indicating an increase in regional cerebral blood flow $(\mathrm{rCBF})$ and $\mathrm{rCBV}$ due to neurovascular coupling (Villringer and Dirnagl, 1995). The post-stimulus undershoot of the BOLD signal was accompanied by a decrease of oxy- $\mathrm{Hb}$ and an increase of deoxy-Hb. Interestingly, a few previous fNIRS studies reported 
Table 1

Changes of oxy-, deoxy-, total hemoglobin ( $\mathrm{Hb})$, cytochrome-c-oxidase (Cyt-Ox), and the blood oxygenation level dependent (BOLD) signal during visual stimulation

\begin{tabular}{|c|c|c|c|}
\hline Method & Chromophore & Stimulus period & Post-stimulus period \\
\hline \multirow[t]{4}{*}{ fNIRS } & $\mathrm{Oxy}-\mathrm{Hb}$ & $235.7 \pm 240.3 \mathrm{nM}^{* * *}$ & $-95.1 \pm 182.6 \mathrm{nM}^{* *}$ \\
\hline & Deoxy-Hb & $-154.5 \pm 87.8 \mathrm{nM}^{* * *}$ & $71.6 \pm 101.7 \mathrm{nM}^{* *}$ \\
\hline & Total $\mathrm{Hb}$ & $81.2 \pm 199.8 \mathrm{nM}^{*}$ & $-23.5 \pm 130.9 \mathrm{nM}$ \\
\hline & Cyt-Ox & $54.1 \pm 58 \mathrm{nM}^{* * *}$ & $5.4 \pm 45.7 \mathrm{nM}$ \\
\hline \multirow[t]{10}{*}{ fMRI } & Averaged BOLD & $1.04 \pm 0.4 \% * * *$ & $-0.23 \pm 0.2 \% * * *$ \\
\hline & $10 \mathrm{~mm}$ & $2.07 \pm 1 \%$ *** & $-0.32 \pm 0.84 \% *$ \\
\hline & $15 \mathrm{~mm}$ & $1.48 \pm 0.54 \% * * *$ & $-0.37 \pm 0.32 \% * * *$ \\
\hline & $20 \mathrm{~mm}$ & $1.23 \pm 0.64 \% * * *$ & $-0.34 \pm 0.25 \% * * *$ \\
\hline & $25 \mathrm{~mm}$ & $1.09 \pm 0.6 \%$ *** & $-0.25 \pm 0.24 \%$ \%** \\
\hline & $30 \mathrm{~mm}$ & $1.04 \pm 0.58 \% * * *$ & $-0.21 \pm 0.19 \%$ \%** \\
\hline & $35 \mathrm{~mm}$ & $0.86 \pm 0.5 \% * * *$ & $-0.2 \pm 0.16 \% * * *$ \\
\hline & $40 \mathrm{~mm}$ & $0.66 \pm 0.36 \% * * *$ & $-0.16 \pm 0.15 \% * * *$ \\
\hline & $45 \mathrm{~mm}$ & $0.35 \pm 0.23 \% * * *$ & $-0.09 \pm 0.14 \% * *$ \\
\hline & $50 \mathrm{~mm}$ & $0.16 \pm 0.18 \% * *$ & $-0.07 \pm 0.15 \% *$ \\
\hline
\end{tabular}

For the BOLD signal, values for each slice are reported beside the averaged value. Distance of the slice to the optode layer is indicated. 1-tailed Student's $t$ test against 0 . Mean differences between vascular response (stimulus period 7 to $13 \mathrm{~s}$, post-stimulus period $19-33 \mathrm{~s}$ after begin of stimulation) and baseline ( -6 to $-1 \mathrm{~s}$ before begin of stimulation).

* $P<0.05$.

** $P<0.01$.

*** $P<0.001$.

similar changes of oxy- and deoxy-Hb during the post-stimulus period. These studies investigated whisker deflection in the rat (Lindauer et al., 2001, 2003) or visual and motor stimulation in humans (Jasdzewski et al., 2003; Schroeter et al., 2004a).

Generally, three mechanisms may contribute to the poststimulus undershoot of the BOLD signal (Aubert and Costalat, 2002; Buxton et al., 1999). First, rCBV may return later to baseline than $\mathrm{rCBF}$ due to delayed venous compliance as proposed by the Balloon and Windkessel models. Second, the undershoot may reflect the persistence of a high oxygen consumption after $\mathrm{rCBF}$ has returned to baseline. Third, an undershoot of the $\mathrm{rCBF}$ could contribute to the BOLD signal undershoot.

In accordance with the Balloon (Buxton et al., 1998) and Windkessel models (Mandeville et al., 1998, 1999), the poststimulus undershoot of the BOLD signal was accompanied by reverse changes of deoxy- $\mathrm{Hb}$, namely, a post-stimulus overshoot of that chromophore in our study. However, there are three inconsistencies with the assumption of a delayed venous compliance. Firstly, in our experiments, total $\mathrm{Hb}$ did not show significant poststimulus changes ${ }^{1}$, although it may be regarded as an equivalent of (corpuscular) rCBV. Second, concentration of oxy-Hb fell below baseline levels during the post-stimulus period, whereas one could expect from the Balloon and Windkessel models values above baseline. Finally, as calculated from results in Table 1, the ratio between post-stimulus and stimulus changes was much higher for oxy- and deoxy- $\mathrm{Hb}(0.4,0.46)$ compared with the averaged BOLD signal $(0.22$; means during the post-stimulus period divided by means during the stimulus period, absolute values). These results

\footnotetext{
${ }^{1}$ One might object that our study missed the post-stimulus elevation of rCBV due to the low signal-to-noise ratio of total $\mathrm{Hb}$ in comparison with oxy- and deoxy-Hb (Table 1, Fig. 3). However, the time course of total $\mathrm{Hb}$ shows during the post-stimulus period values slightly below baseline, indicating that increasing the signal-to-noise ratio will not show a poststimulus elevation of rCBV.
}

indicate relatively higher post-stimulus effects for the optical parameters.

As illustrated in Fig. 1, one can assume that changes of the optical and BOLD signals originated from similar macroscopic brain regions in our study. However, fNIRS and fMRI are sensitive to different vascular compartments. It has been suggested that optical methods, such as fNIRS, are sensitive to the microvasculature, namely, to arterioles, capillaries, and venules (Boushel et al., 2001; Cannestra et al., 2001; Schroeter et al., 2004b), because, for larger vessels such as arteries and veins, entering light cannot escape (Liu et al., 1995a,b). Recently, Lu et al. (2003, 2004) showed by vascular space occupancy dependent fMRI that, in the human brain, rCBV returned immediately after visual stimulation to baseline values if the signal's origin was limited to the microvasculature. Jasdzewski et al. (2003) reported similar results for total $\mathrm{Hb}$ after visual and motor stimulation in humans. These results fit very well with our data.

During the post-stimulus period, we observed a significant undershoot of oxy-Hb (beside the overshoot of deoxy-Hb) that cannot be explained by a delayed return of $\mathrm{rCBV}$ to baseline levels. Hence, the post-stimulus changes of oxy- and deoxy-Hb may reflect the persistence of a high-level oxygen consumption after rCBF has returned to baseline (Aubert and Costalat, 2002; Buxton et al., 1999; Frahm et al., 1996). A high oxygen consumption diminishes the BOLD signal and concentrations of oxy-Hb and elevates deoxy- $\mathrm{Hb}$ without influencing total $\mathrm{Hb}$. Therefore, it can explain the post-stimulus events. This argument is strongly supported by a recent multimodal fMRI study (Lu et al., 2003, 2004). Investigating visual stimulation in humans, the authors reported that the long post-stimulus undershoot of the BOLD signal of approximately $30 \mathrm{~s}$, such as in our study, was accompanied by a prolonged elevation of oxygen utilization with the same time course. Microvascular $\mathrm{rCBV}$, in contrast, returned immediately after stimulation to baseline values (see above).

Lastly, it needs an explanation why the post-stimulus effects (as measured by the ratio between post-stimulus and stimulus changes) were relatively stronger for the optical parameters in comparison with the BOLD signal in our study. Obviously, oxygen is consumed mainly in capillaries. If optical methods are particularly sensitive to the microvasculature (Boushel et al., 2001; Cannestra et al., 2001; Liu et al., 1995a,b; Schroeter et al., 2004b) whereas the BOLD signal represents all spatial scales of venous vessels (Lee et al., 2001; Strangman et al., 2002), a prolonged poststimulus elevation of oxygen utilization would be more visible in the optical parameters. A recent study found a negligible signal attenuation of the BOLD signal by diffusion weighting at $3.0 \mathrm{~T}$ during the post-stimulus undershoot, supporting the assumption of an extravascular origin of the BOLD post-stimulus undershoot (Mildner et al., 2001). Results suggest that the post-stimulus undershoot of the BOLD signal is related to an elevated vessel volume as suggested by the Balloon and Windkessel models and excludes an increase in the concentration of deoxy-Hb in postcapillary venous vessels. Jones (1999) measured changes in the relaxation times $\mathrm{R}_{2}$ and $\mathrm{R}_{2} *$ at $1.5 \mathrm{~T}$ during the post-stimulus period of the BOLD signal. Jones et al. concluded that intravascular signals, if present in this period, stem from small vessels (not affected by diffusion weighting). Although this fits well with our assumptions, the elevation in the concentration of deoxy-Hb by an increased oxygen consumption in capillaries without affecting the concentration of deoxy-Hb in post-capillary veins remains to be explained. 

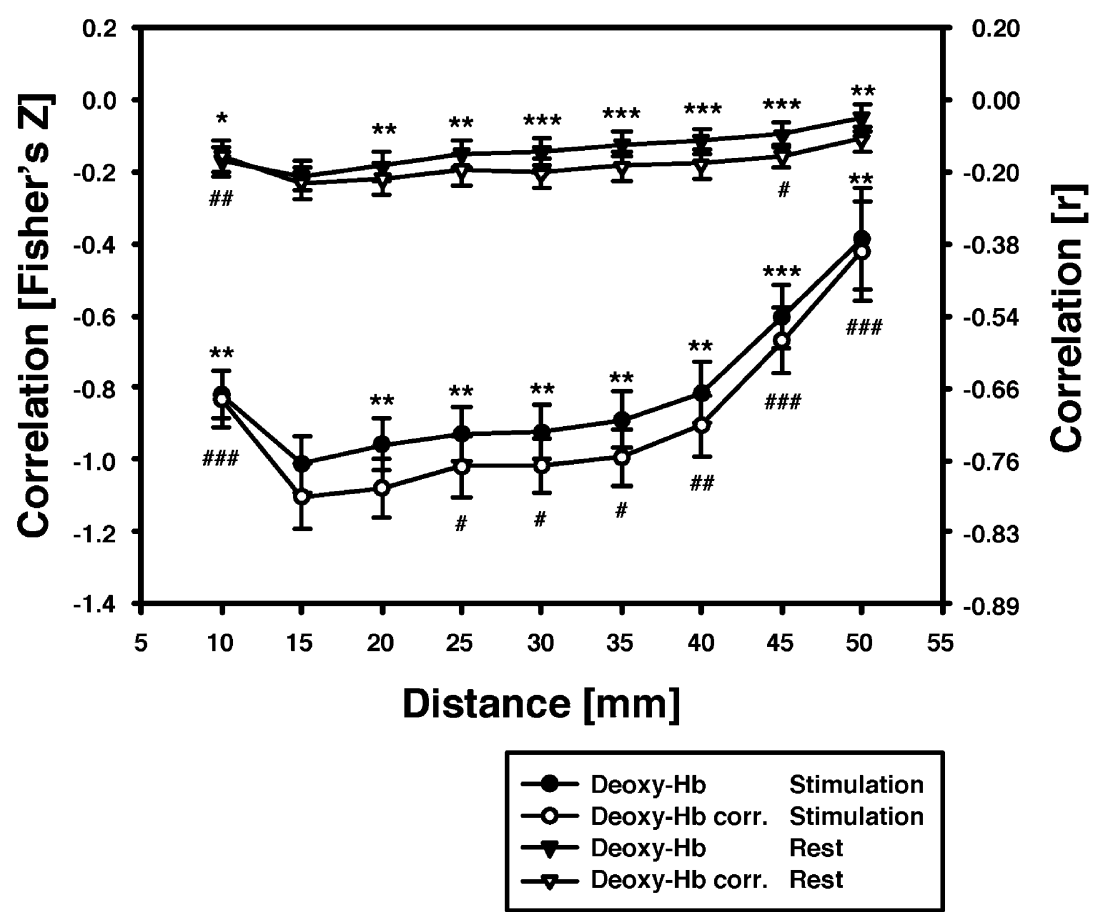

Fig. 4. Depth penetration of fNIRS. Time courses of deoxy-hemoglobin $(\mathrm{Hb})$ as measured by fNIRS were correlated with the BOLD signal as measured by fMRI for every slice separately. Correlation is shown for visual stimulation and rest (closed eyes). Pearson correlation coefficients and partial correlation coefficients (adjusted for changes in total Hb; corr.) were normalized with a Fisher's $Z$ transformation. Distance to optode layer. Mean \pm SEM. $* * * \# \# P<$ $0.001, * * \# P<0.01, * \# P<0.05$ 2-tailed paired Student's $t$ test vs. values at $15 \mathrm{~mm}$.

The post-stimulus changes of oxy- and deoxy-Hb may also be explained by a reduced post-stimulus rCBF (Aubert and Costalat, 2002; Buxton et al., 1999), which is possibly preceded by an inhibition of neural activity after stimulation (Logothetis et al., 2001; Wenzel et al., 2000). Our study did not measure simultaneously changes in $\mathrm{rCBF}$, which should be done in future experiments. The post-stimulus undershoot of rCBF occurs only in a minority of subjects, is highly variable (Irikura et al., 1994; Ma et al., 1996), task-dependent (Hoge et al., 1999), and can even be explained as an artifact of delayed rCBV changes (Mandeville et al., 1999). Furthermore, the ratio between post-stimulus and stimulus changes of $\mathrm{rCBF}$, as calculated from these studies, reaches only values between approximately 0.15 and 0.3 (Hoge et al. 1999; Irikura et al., 1994; Ma et al., 1996). Hence, it cannot explain the fNIRS data of our study. One can conclude that the above mechanisms cannot be the main source for the post-stimulus changes.

In summary, our study is the first one that investigated the poststimulus period by both fNIRS and fMRI. This multimodal approach investigates simultaneously events in the microvascular

Table 2

Correlation between the blood oxygenation level dependent (BOLD) signal and oxy-, deoxy-, total hemoglobin (Hb), and cytochrome-c-oxidase (Cyt-Ox) during visual stimulation

\begin{tabular}{|c|c|c|c|c|c|c|c|c|}
\hline \multirow[t]{2}{*}{ Subject } & \multicolumn{4}{|c|}{$\underline{\text { Stimulus period }}$} & \multicolumn{4}{|c|}{ Post-stimulus period } \\
\hline & $\mathrm{Oxy}-\mathrm{Hb}$ & Deoxy-Hb & Total $\mathrm{Hb}$ & Cyt-Ox & Oxy-Hb & Deoxy-Hb & Total $\mathrm{Hb}$ & Cyt-Ox \\
\hline 1 & 1.31 & -1.33 & 0.87 & 0.41 & 1.11 & -0.89 & 0.62 & 0.02 \\
\hline 2 & 0.73 & -0.87 & -0.14 & 0.56 & 0.20 & -0.50 & -0.02 & 0.17 \\
\hline 3 & 0.27 & -0.41 & 0.03 & 0.05 & -0.11 & 0.02 & -0.09 & 0.05 \\
\hline 4 & 0.86 & -1.23 & 0.11 & 0.86 & -0.40 & -0.29 & -0.46 & 0.23 \\
\hline 5 & 0.36 & -0.71 & -0.19 & 0.39 & 0.19 & -0.14 & 0.10 & 0.07 \\
\hline 6 & 1.31 & -1.01 & 0.68 & 0.23 & 0.39 & -0.66 & -0.15 & 0.13 \\
\hline 7 & 0.30 & -0.62 & -0.09 & 0.26 & -0.41 & -0.37 & -0.45 & -0.09 \\
\hline 8 & 0.32 & -1.35 & -0.28 & 0.95 & 0.70 & -0.39 & 0.52 & -0.15 \\
\hline 9 & 0.31 & -0.95 & -0.19 & 0.28 & 0.28 & -0.01 & 0.15 & -0.27 \\
\hline 10 & -0.23 & -0.97 & -0.54 & 0.55 & 0.13 & -0.31 & -0.01 & -0.05 \\
\hline 11 & 0.63 & -1.27 & 0.07 & 0.19 & -0.69 & -0.31 & -0.78 & 0.27 \\
\hline 12 & 1.46 & -1.26 & 0.54 & 1.38 & 0.55 & -0.46 & 0.13 & 0.43 \\
\hline Mean \pm SD & $0.64 \pm 0.52$ & $-1 \pm 0.3$ & $0.07 \pm 0.42$ & $0.51 \pm 0.38$ & $0.16 \pm 0.51$ & $-0.36 \pm 0.26$ & $-0.04 \pm 0.4$ & $0.07 \pm 0.19$ \\
\hline
\end{tabular}

Analysis was performed for the slice $15 \mathrm{~mm}$ below the optode layer. Correlation between the BOLD signal and the chromophores was performed separately for the stimulus period $(0-15 \mathrm{~s})$ and the post-stimulus period ( $15-48 \mathrm{~s}$ after stimulation onset). Pearson correlation coefficients were normalized with a Fisher's $Z$ transformation. 
(fNIRS) and the post-capillary venous compartment (BOLD fMRI). Results suggest that the post-stimulus events as measured by fNIRS are dominated by a prolonged high-level oxygen consumption in the microvasculature. The contribution of a delayed return of $\mathrm{rCBV}$ to the BOLD post-stimulus undershoot in post-capillary veins as suggested by the Balloon and Windkessel models remains ambiguous.

During visual stimulation, we found the strongest correlation between the BOLD signal and deoxy-Hb, which was highest in a depth of $15 \mathrm{~mm}$ beneath the skin. Oxy-, total $\mathrm{Hb}$, and Cyt-Ox were correlated with the BOLD signal as well, although with smaller correlation coefficients. Our data agree well with Ogawa et al. (1993) and Buxton et al. (1998), which assume that the BOLD signal arises mainly from magnetic disturbances caused by the paramagnetic deoxy-Hb. Our data are also consistent with simultaneous fMRI and fNIRS experiments showing a high correlation between the BOLD signal and deoxy-Hb (Kida et al., 1996; Mehagnoul-Schipper et al., 2002; Punwani et al., 1997, 1998; Toronov et al., 2003) and with Pouratian et al. (2002) who showed a high temporal and spatial correlation between the BOLD signal and optical intrinsic signals measured at $610 \mathrm{~nm}$ (particularly sensitive to deoxy-Hb) in humans. Differences to Strangman et al. (2002), who found a lower correlation of the BOLD signal with deoxy- compared with oxy-Hb during finger flexion/ extension motor activation, may be explained by their less (three) subjects, different (block) design, their shorter emitter-detector separation (Germon et al., 1999), and non-linear changes of oxy$\mathrm{Hb}$ in relation to deoxy-Hb in the motor cortex (Wolf et al., 2002). Because of the known linear relationship between changes of oxyand deoxy-Hb in the visual cortex (Fig. 3; Wolf et al., 2002), we decided to apply a visual paradigm in contrast to their study.

Crucially, we investigated for the first time simultaneously the BOLD signal and changes of fNIRS chromophores during rest and found that deoxy-Hb was again tightly related to the BOLD signal. This analysis covered un-averaged data and was independent of functional stimulation that may bias results. Correlation during rest is presumably based on spontaneous vascular oscillations related to vasomotion, such as low and very low frequency oscillations and on oscillations related to respiration (Colantuoni et al., 1994; Obrig et al., 2000a; Schroeter et al., 2004b, 2005). With an effective sampling rate of $1 \mathrm{~Hz}$, oscillations due to heart pulsations did not influence our measurements.

We found the highest correlation between deoxy-Hb and the BOLD signal in a depth of $1.5 \mathrm{~cm}$. One may conclude that fNIRS covers the outer $1 \mathrm{~cm}$ of the brain cortex, which is in good accordance with two simultaneous fNIRS $/ \mathrm{H}_{2}^{15} \mathrm{O}$-PET studies measuring the hemodynamic response in the parietal cortex of patients with Alzheimer's disease during a Stroop task (Hock et al., 1997) and in the forehead of healthy elderly subjects during calculation and a Stroop task (Villringer et al., 1997). It is well known that the hemodynamic response declines in associative cortices with aging (Schroeter et al., 2003, 2004b). Hence, aging effects may have biased the results of both PET studies in contrast to our study.

\section{Conclusion}

Our study investigated for the first time the post-stimulus undershoot in a simultaneous fMRI/fNIRS study in humans during visual stimulation. Results suggest that the post-stimulus events as measured by fNIRS are dominated by a prolonged high-level oxygen consumption in the microvasculature. The contribution of a delayed return of blood volume to the BOLD post-stimulus undershoot in post-capillary veins as suggested by the Balloon and Windkessel models remains ambiguous. Temporal changes in the BOLD signal were highly correlated with deoxy-Hb, with lower correlation values for oxy- and total $\mathrm{Hb}$. Furthermore, data show that fNIRS covers the outer $1 \mathrm{~cm}$ of the brain cortex.

\section{References}

Aubert, A., Costalat, R., 2002. A model of the coupling between brain electrical activity, metabolism, and hemodynamics: application to the interpretation of functional neuroimaging. NeuroImage 17, 1162-1181.

Bortz, J., 1999. Statistik für Sozialwissenschaftler, 5th ed. Springer, Berlin.

Boushel, R., Langberg, H., Olesen, J., Gonzales-Alonzo, J., Bülow, J., Kjaer, M., 2001. Monitoring tissue oxygen availability with nearinfrared spectroscopy (NIRS) in health and disease. Scand. J. Med. Sci. Sports 11, 213-222.

Burock, M.A., Buckner, R.L., Woldorff, M.G., Rosen, B.R., Dale, A.M., 1998. Randomized event-related experimental designs allow for extremely rapid presentation rates using functional MRI. NeuroReport 9, 3735-3739.

Buxton, R.B., Wong, E.C., Frank, L.R., 1998. Dynamics of blood flow and oxygenation changes during brain activation: the balloon model. Magn. Reson. Med. 39, 855-864.

Buxton, R.B., Wong, E.C., Frank, L.R., 1999. The post-stimulus undershoot of the functional MRI signal. In: Moonen, C.T.W., Bandettini, P.A. (Eds.), Functional MRI. Springer, Berlin, pp. 253-262.

Cannestra, A.F., Pouratian, N., Bookheimer, S.Y., Martin, N.A., Becker, D.P., Toga, A.W., 2001. Temporal spatial differences observed by functional MRI and human intraoperative optical imaging. Cereb. Cortex 11, 773-782.

Colantuoni, A., Bertuglia, S., Intaglietta, M., 1994. Microvascular vasomotion-Origin of laser Doppler flux motion. Int. J. Microcirc. Clin. Exp. 14, 151-158.

Cope, M., Delpy, D.T., 1988. System for long-term measurement of cerebral blood and tissue oxygenation on newborn infants by near infrared transillumination. Med. Biol. Eng. Comput. 26, 289-294.

Duncan, A., Meek, J.H., Clemence, M., Elwell, C.E., Tyszczuk, L., Cope, M., Delpy, D.T., 1995. Optical pathlength measurements on adult head, calf and forearm and the head of the newborn infant using phase resolved optical spectroscopy. Phys. Med. Biol. 40, 295-304.

Frahm, J., Krüger, G., Merboldt, K.D., Kleinschmidt, A., 1996. Dynamic uncoupling and recoupling of perfusion and oxidative metabolism during focal brain activation in man. Magn. Reson. Med. 35, 143-148.

Germon, T.J., Evans, P.D., Barnett, N.J., Wall, P., Manara, A.R., Nelson, R.J., 1999. Cerebral near infrared spectroscopy: emitter-detector separation must be increased. Br. J. Anaesth. 82, 831-837.

Heekeren, H.R., Kohl, M., Obrig, H., Wenzel, R., von Pannwitz, W., Matcher, S.J., Dirnagl, U., Cooper, C.E., Villringer, A., 1999. Noninvasive assessment of changes in cytochrome-c oxidase oxidation in human subjects during visual stimulation. J. Cereb. Blood Flow Metab. 19, 592-603.

Hock, C., Villringer, K., Muller-Spahn, F., Wenzel, R., Heekeren, H., Schuh-Hofer, S., Hofmann, M., Minoshima, S., Schwaiger, M., Dirnagl, U., Villringer, A., 1997. Decrease in parietal cerebral hemoglobin oxygenation during performance of a verbal fluency task in patients with Alzheimer's disease monitored by means of near-infrared spectroscopy (NIRS) - Correlation with simultaneous rCBF-PET measurements. Brain Res. 755, 293-303.

Hoge, R.D., Atkinson, J., Gill, B., Crelier, G.R., Marrett, S., Pike, G.B., 1999. Stimulus-dependent BOLD and perfusion dynamics in human V1. NeuroImage 9, 573-585. 
Hoge, R.D., Franceschini, M.A., Covolan, R.J.M., Huppert, T., Mandeville, J.B., Boas, D.A., 2005. Simultaneous recording of task-induced changes in blood oxygenation, volume, and flow using diffuse optical imaging and arterial spin-labeling MRI. NeuroImage 25, 701-707.

Homan, R.W., Herman, J., Purdy, P., 1987. Cerebral location of international 10-20 system electrode placement. Electroencephalogr. Clin. Neurophysiol. 66, 376-381.

Hoshi, Y., 2003. Functional near-infrared optical imaging: utility and limitations in human brain mapping. Psychophysiology 40, 511-520.

Irikura, K., Maynard, K.I., Moskowitz, M.A., 1994. Importance of nitric oxide synthase inhibition to the attenuated vascular responses induced by topical 1-nitroarginine during vibrissal stimulation. J. Cereb. Blood Flow Metab. 14, 45-48.

Jasdzewski, G., Strangman, G., Wagner, J., Kwong, K.K., Poldrack, R.A., Boas, D.A., 2003. Differences in the hemodynamic response to eventrelated motor and visual paradigms as measured by near-infrared spectroscopy. NeuroImage 20, 479-488.

Jones, R.A., 1999. Origin of the signal undershoot in BOLD studies of the visual cortex. NMR Biomed. 12, 299-308.

Kennan, R.P., Kim, D., Maki, A., Koizumi, H., Constable, R.T., 2002. Noninvasive assessment of language lateralization by transcranial near infrared optical topography and functional MRI. Hum. Brain Mapp. 16, $183-189$.

Kida, I., Yamamoto, T., Tamura, M., 1996. Interpretation of BOLD MRI signals in rat brain using simultaneously measured near-infrared spectrophotometric information. NMR Biomed. 9, 333-338.

Kleinschmidt, A., Obrig, H., Requardt, M., Merboldt, K.D., Dirnagl, U., Villringer, A., Frahm, J., 1996. Simultaneous recording of cerebral blood oxygenation changes during human brain activation by magnetic resonance imaging and near-infrared spectroscopy. J. Cereb. Blood Flow Metab. 16, 817-826.

Lee, J.H., Garwood, M., Menon, R., Adriany, G., Andersen, P., Truwit, C.L., Ugurbil, K., 1995. High contrast and fast three-dimensional magnetic resonance imaging at high fields. Magn. Reson. Med. 34, $308-312$.

Lee, S.P., Duong, T.Q., Yang, G., Iadecola, C., Kim, S.G., 2001. Relative changes of cerebral arterial and venous blood volumes during increased cerebral blood flow: implications for BOLD fMRI. Magn. Reson. Med. $45,791-800$.

Lindauer, U., Royl, G., Leithner, C., Kühl, M., Gold, L., Gethmann, J., Kohl-Bareis, M., Villringer, A., Dirnagl, U., 2001. No evidence for early decrease in blood oxygenation in rat whisker cortex in response to functional activation. NeuroImage 13, 988-1001.

Lindauer, U., Gethmann, J., Kühl, M., Kohl-Bareis, M., Dirnagl, U., 2003. Neuronal activity-induced changes of local cerebral microvascular blood oxygenation in the rat: effect of systemic hyperoxia or hypoxia. Brain Res. 975, 135-140.

Liu, H., Boas, D.A., Zhang, Y., Yodh, A.G., Chance, B., 1995a. Determination of optical properties and blood oxygenation in tissue using continuous NIR light. Phys. Med. Biol. 40, 1983-1993.

Liu, H., Chance, B., Hielscher, A.H., Jacques, S.L., Tittel, F.K., 1995 b. Influence of blood vessels on the measurement of hemoglobin oxygenation as determined by time-resolved reflectance spectroscopy. Med. Phys. 22, $1209-1217$.

Logothetis, N.K., Pauls, J., Augath, M., Trinath, T., Oeltermann, A., 2001. Neurophysiological investigation of the basis of the fMRI signal. Nature $412,150-157$.

Lohmann, G., Mueller, K., Bosch, V., Mentzel, H., Hessler, S., Chen, L., von Cramon, D.Y., 2000. LIPSIA-Leipzig image processing and statistical inference algorithms. Technical Report. Max Planck Institute of Cognitive Neuroscience, Leipzig.

Lu, H.Z., Golay, X., Pekar, J.J., van Zijll, P.C.M., 2003. Functional magnetic resonance imaging based on changes in vascular space occupancy. Magn. Reson. Med. 50, 263-274.

Lu, H.Z., Golay, X., Pekar, J.J., van Zijll, P.C.M., 2004. Sustained poststimulus elevation in cerebral oxygen utilization after vascular recovery. J. Cereb. Blood Flow Metab. 24, 764-770.
Ma, J., Ayata, C., Huang, P.L., Fishman, M.C., Moskowitz, M.A., 1996. Regional cerebral blood flow response to vibrissal stimulation in mice lacking type I NOS gene expression. Am. J. Physiol. 270, H1085-H1090.

Mandeville, J.B., Marota, J.J.A., Kosofsky, B.E., Keltner, J.R., Weissleder, R., Rosen, B.R., Weisskoff, R.M., 1998. Dynamic functional imaging of relative cerebral blood volume during rat forepaw stimulation. Magn. Reson. Med. 39, 615-624.

Mandeville, J.B., Marota, J.J.A., Ayata, C., Zaharchuk, G., Moskowitz, M.A., Rosen, B.R., Weisskoff, R.M., 1999. Evidence of a cerebrovascular postarteriole windkessel with delayed compliance. J. Cereb. Blood Flow Metab. 19, 679-689.

Mehagnoul-Schipper, D.J., van der Kallen, B.F., Colier, W.N., van der Sluijs, M.C., van Erning, L.J., Thijssen, H.O., Oeseburg, B., Hoefnagels, W.H., Jansen, R.W., 2002. Simultaneous measurements of cerebral oxygenation changes during brain activation by near-infrared spectroscopy and functional magnetic resonance imaging in healthy young and elderly subjects. Hum. Brain Mapp. 16, 14-23.

Mildner, T., Norris, D.G., Schwarzbauer, C., Wiggins, C.J., 2001. A qualitative test of the balloon model for BOLD-based MR signal changes at 3T. Magn. Reson. Med. 46, 891-899.

Norris, D.G., 2000. Reduced power multi-slice MDEFT imaging. J. Magn. Reson. Imaging 11, 445-451.

Obrig, H., Neufang, M., Wenzel, R., Kohl, M., Steinbrink, J., Einhäupl, K., Villringer, A., 2000a. Spontaneous low frequency oscillations of cerebral hemodynamics and metabolism in human adults. NeuroImage $12,623-639$.

Obrig, H., Wenzel, R., Kohl, M., Horst, S., Wobst, P., Steinbrink, J., Thomas, F., Villringer, A., 2000b. Near-infrared spectroscopy: does it function in functional activation studies of the adult brain? Int. J. Psychophysiol. 35, 125-142.

Ogawa, S., Menon, R.S., Tank, D.W., Kim, S.G., Merkle, H., Ellermann, J.M., Ugurbil, K., 1993. Functional brain mapping by blood oxygenation level-dependent contrast magnetic resonance imaging: a comparison of signal characteristics with a biophysical model. Biophys. J. 64, $803-812$

Okamoto, M., Dan, H., Sakamoto, K., Takeo, K., Shimizu, K., Kohno, S., Oda, I., Isobe, S., Suzuki, T., Kohyama, K., Dan, I., 2004. Threedimensional probabilistic anatomical cranio-cerebral correlation via the international 10-20 system oriented for transcranial functional brain mapping. NeuroImage 21, 99-111.

Pouratian, N., Sicotte, N., Rex, D., Martin, N.A., Becker, D., Cannestra, A.F., Toga, A.W., 2002. Spatial/temporal correlation of BOLD and optical intrinsic signals in humans. Magn. Reson. Med. 47, 766-776.

Punwani, S., Cooper, C.E., Clemence, M., Penrice, J., Amess, P., Thorton, J., Ordidge, R.J., 1997. Correlation between absolute deoxyhaemoglobin $[\mathrm{dHb}]$ measured by near infrared spectroscopy (NIRS) and absolute $\mathrm{R} 2^{\prime}$ as determined by magnetic resonance imaging (MRI). Adv. Exp. Med. Biol. 413, 129-137.

Punwani, S., Ordidge, R.J., Cooper, C.E., Amess, P., Clemence, M., 1998. MRI measurements of cerebral deoxyhaemoglobin concentration $[\mathrm{dHb}]$ - Correlation with near infrared spectroscopy (NIRS). NMR Biomed. 11, 281-289.

Schroeter, M.L., Zysset, S., Kupka, T., Kruggel, F., von Cramon, D.Y., 2002. Near-infrared spectroscopy can detect brain activity during a color-word matching Stroop task in an event-related design. Hum. Brain Mapp. 17, 61-71.

Schroeter, M.L., Zysset, S., Kruggel, F., von Cramon, D.Y., 2003. Agedependency of the hemodynamic response as measured by functional near-infrared spectroscopy. NeuroImage 19, 555-564.

Schroeter, M.L., Bücheler, M.M., Müller, K., Uludag, K., Obrig, H., Lohmann, G., Tittgemeyer, M., Villringer, A., von Cramon, D.Y., 2004a. Towards a standard analysis for functional near-infrared imaging. NeuroImage 21, 283-290.

Schroeter, M.L., Schmiedel, O., von Cramon, D.Y., 2004b. Spontaneous low frequency oscillations decline in the aging brain. J. Cereb. Blood Flow Metab. 24, 1183-1191. 
Schroeter, M.L., Zysset, S., von Cramon, D.Y., 2004c. Shortening intertrial intervals in event-related cognitive studies with near-infrared spectroscopy. NeuroImage 22, 341-346.

Schroeter, M.L., Zysset, S., Wahl, M.M., von Cramon, D.Y., 2004d. Prefrontal activation due to Stroop interference increases during development-An event-related fNIRS study. NeuroImage 23, $1317-1325$.

Schroeter, M.L., Bücheler, M.M., Preul, C., Scheid, R., Schmiedel, O., Guthke, T., von Cramon, D.Y., 2005. Spontaneous slow hemodynamic oscillations are impaired in cerebral microangiopathy. J. Cereb. Blood Flow Metab. (published online June 1).

Seiyama, A., Seki, J., Tanabe, H.C., Sase, I., Takatsuki, A., Miyauchi, S., Eda, H., Hayashi, S., Imaruoka, T., Iwakura, T., Yanagida, T., 2004. Circulatory basis of fMRI signals: relationship between changes in the hemodynamic parameters and BOLD signal intensity. NeuroImage 21, $1204-1214$.

Siegel, A.M., Culver, J.P., Mandeville, J.B., Boas, D.A., 2003. Temporal comparison of functional brain imaging with diffuse optical tomography and fMRI during rat forepaw stimulation. Phys. Med. Biol. 48, $1391-1403$.

Strangman, G., Culver, J.P., Thompson, J.H., Boas, D.A., 2002. A quantitative comparison of simultaneous BOLD fMRI and NIRS recordings during functional brain activation. NeuroImage 17, $719-731$.

Thevenaz, P., Blu, T., Unser, M., 2000. Image Interpolation and resampling. In: Bankman, I.N. (Ed.), Handbook of Medical Image Processing and Analysis. Academic Press, San Diego, pp. 393-420.

Toronov, V., Webb, A., Choi, J.H., Wolf, M., Michalos, A., Gratton, E., Hueber, D., 2001a. Investigation of human brain hemodynamics by simultaneous near-infrared spectroscopy and functional magnetic resonance imaging. Med. Phys. 28, 521-527.

Toronov, V., Webb, A., Choi, J.H., Wolf, M., Safonova, L., Wolf, U., Gratton, E., 2001b. Study of local cerebral hemodynamics by frequency-domain near-infrared spectroscopy and correlation with simultaneously acquired functional magnetic resonance imaging. Opt. Express 9, 417-427.
Toronov, V., Walker, S., Gupta, R., Choi, J.H., Gratton, E., Hueber, D., Webb, A., 2003. The roles of changes in deoxyhemoglobin concentration and regional cerebral blood volume in the fMRI BOLD signal. NeuroImage 19, 1521-1531.

Ugurbil, K., Garwood, M., Ellermann, J., Hendrich, K., Hinke, R., Hu, X., Kim, S.G., Menon, R., Merkle, H., Ogawa, S., Salmi, R., 1993. Imaging at high magnetic fields: initial experiences at 4T. Magn. Reson. Q. 9, $259-277$.

Uludag, K., Kohl, M., Steinbrink, J., Obrig, H., Villringer, A., 2002. Cross talk in the Lambert-Beer calculation for near-infrared wavelengths estimated by Monte Carlo simulations. J. Biomed. Opt. 7, 51-59.

Villringer, A., Chance, B., 1997. Non-invasive optical spectroscopy and imaging of human brain function. Trends Neurosci. 20, 435-442.

Villringer, A., Dirnagl, U., 1995. Coupling of brain activity and cerebral blood flow: basis of functional neuroimaging. Cerebrovasc. Brain Metab. Rev. 7, 240-276.

Villringer, K., Minoshima, S., Hock, C., Obrig, H., Ziegler, S., Dirnagl, U., Schwaiger, M., Villringer, A., 1997. Assessment of local brain activation. A simultaneous PET and near-infrared spectroscopy study. Adv. Exp. Med. Biol. 413, 149-153.

Weisskoff, R.M., 1999. Basic theoretical models of BOLD signal change. In: Moonen, C.T.W., Bandettini, P.A. (Eds.), Functional MRI. Springer, Berlin, pp. 115-124.

Wenzel, R., Wobst, P., Heekeren, H.H., Kwong, K.K., Brandt, S.A., Kohl, M., Obrig, H., Dirnagl, U., Villringer, A., 2000. Saccadic suppression induces focal hypooxygenation in the occipital cortex. J. Cereb. Blood Flow Metab. 20, 1103-1110.

Wobst, P., Wenzel, R., Kohl, M., Obrig, H., Villringer, A., 2001. Linear aspects of changes in deoxygenated hemoglobin concentration and cytochrome oxidase oxidation during brain activation. NeuroImage 13, $520-530$.

Wolf, M., Wolf, U., Toronov, V., Michalos, A., Paunescu, L.A., Choi, J.H., Gratton, E., 2002. Different time evolution of oxy- and deoxyhemoglobin concentration changes in the visual and motor cortices during functional stimulation: a near-infrared spectroscopy study. NeuroImage $16,704-712$. 